\title{
Análisis de la tecnología lítica del sitio Villavil localizado en el valle de Hualfín (Provincia de Catamarca, Argentina)
}

\author{
Julieta LYNCH y Virginia LYNCH \\ División Arqueología, Museo de La Plata, FCNyM, UNPL-CONICET \\ julietalynch@yahoo.es lynchvirginia@gmail.com
}

Recibido: $23 / 2 / 2015$

Aceptado: $18 / 2 / 2016$

\begin{abstract}
RESUMEN
El siguiente trabajo tiene por objetivo profundizar el conocimiento acerca de la producción de la tecnológica lítica de los grupos que habitaron el sitio Villavil, ubicado al norte del valle de Hualfín, Catamarca, Argentina. De este sitio se han obtenido dataciones radiocarbónicas que se ubican desde la transición del período Formativo Temprano/Medio (500-900/1000 d.C.) hasta momentos incaicos (1430 d.C.), y han podido ser recuperados abundantes materiales arqueológicos tanto en superficie como en estratigrafía. Los resultados obtenidos a partir del análisis lítico realizado de materiales en estratigrafía, han permitido identificar una baja producción de instrumentos formatizados y tecnología expeditiva, realizados a partir de materias primas disponibles en las inmediaciones del sitio. Esto permitiría sostener la hipótesis de una tendencia hacia la minimización del tiempo invertido en las distintas etapas de producción de la tecnología lítica, generada y utilizada por los grupos que habitaron Villavil en momentos tardíos.
\end{abstract}

Palabras clave: Valle de Hualfín, período Tardío, tecnología lítica, sitio Villavil

\section{Analysis of the Lithic Technology of Villavil Site, Hualfin Valley (Province of Catamarca, Argentina)}

\begin{abstract}
This paper aims to increase the knowledge of the lithic technology of the groups in Villavil site, Hualfin Valley, Catamarca, Argentina. From this site has been obtained radiocarbon dates from transition Formative Early/ Middle Period (500-900/1000 d.C.) to Inca times (1430 d.C.), and have been recovered several archaeological materials on surface and in stratigraphy. The results obtained from the lithic analysis on stratigraphy materials have identified a low production of formatized instrument and expeditive technology, made from local raw material located around the site. This study suggested a minimization in the invested time on the different production stages of the lithic technology generated and used by the Villavil lates societies.
\end{abstract}

Key words: Hualfín Valley, Late Period, Lithic technology, Villavil site

Sumario: 1. Introducción. 2. Microambiente y recursos líticos disponibles. 3. Sitio Villavil: características principales. 4. Metodología aplicada. 5. Análisis del material lítico recuperado en el Recinto Rectangular 1. 6. Discusión y conclusiones. 7. Referencias bibliográficas.

\section{Introducción}

En Argentina, los estudios específicos sobre material lítico han estado focalizados mayormente en el estudio de grupos de cazadores-recolectores. Sin embargo, en los últimos años los trabajos sobre el material lítico recuperado de sitios asignados a momentos más tardíos están siendo cada vez más numerosos para el Noroeste Argentino (NOA). Las investigaciones realizadas hasta el momento por distintos investigadores en esta región de la Argentina, han contribuido enormemente a la comprensión de los procesos de producción y utilización de herramientas líticas para ocupaciones tardías 
(Ávalos 1998, 2002; Ávalos y Chaparro 2008; Chaparro 2002, 2009, 2013; Chaparro y Ávalos 2014; Elías 2005, 2007, 2008; Ledesma 2003; Ratto 2003; Ratto y Williams 1995; Sprovieri 2005, 2006; Sprovieri y Baldini 2007). Estas investigaciones están focalizadas en diferentes regiones del Noroeste Argentino como por ejemplo la zona de puna (Quebrada de Humahuaca), y el valle Calchaquí medio y norte. Si bien existen ciertas particularidades locales, estos estudios han permitido caracterizar la producción lítica básicamente como ubicua y generalizada, sin una marcada división de las etapas de reducción, asociada a contextos domésticos de procesamiento y consumo. También se destaca en estos estudios la presencia de herramientas líticas asociadas a otras tecnologías, como las agrícolas, de recolección o de laboreo de la tierra, de molienda y metalúrgicas, muchas de las cuales no requieren manufactura. A su vez, estos estudios identifican el empleo de las mismas fuentes de obsidiana que se explotaban desde períodos previos (Escola 2000; Elías y Escola 2010; Yacobaccio et al. 2004).

Ahora bien, considerando la tecnología en un sentido amplio, como componente dinámico de un sistema mayor, interrelacionado con otros dominios (económico, ideológico, político, social [Nelson 1991, 1996; Torrence 1989]), vemos que los cambios en las actividades de subsistencia para momentos tardíos habrían implicado otros en las estrategias tecnológicas de las sociedades de nuestro interés; tal es el caso del tiempo destinado a la producción de diferentes tecnologías (cerámica, lítica, de irrigación, de almacenaje) en función de su grado de compromiso con la subsistencia (Elías y Escola 2010; Olivera y Vigliani 2000-02).

Es así que, desde $c a$. $2000 \mathrm{AP}$, la agricultura habría adquirido mayor importancia en la subsistencia (Olivera y Podestá 1993), condiciones que se incrementan hacia la Puna Meridional Argentina en donde la concentración de nutrientes se da en espacios muy restringidos en un marco desértico de extrema aridez (Yacobaccio 1994).

Hacia $c a$. 1000 AP, con la llegada de los incas a la microrregión, este proceso fue acompañado por un aumento del sedentarismo en relación con el incremento gradual de la presión poblacional y de la agricultura; el aumento poblacional y el consecuente incremento de la producción de diferentes cultivos, habrían generado un mayor sedentarismo y una reducción en la movilidad, modificando la organización social y política de estos grupos y provocando, a su vez, una mayor concentración y burocratización del poder y de sus asentamientos (Olivera y Vigliani 2000-02, citado en Elías 2012). La mayor diversificación de actividades relacionadas con la agricultura llevaron a la acentuación, durante momentos más tardíos, de la importancia del componente expeditivo, ya señalado para momentos formativos por Escola $(2000,2004)$.

En la zona del valle de Hualfín, en la provincia de Catamarca, han sido realizados algunos trabajos presentando evidencias de la producción lítica durante el período de Desarrollos Regionales (900/1000-1430 d.C.) y la posterior ocupación incaica. En estas investigaciones se ve una continuidad en la utilización de la materia prima local, como el cuarzo (Flores 2012) y el basalto, y la incorporación de otros materiales como el xilópalo y la obsidiana proveniente de lugares más alejados de la región, probablemente de la zona puneña de la provincia (Flores y Wynveldt 2009; Lynch y Lynch 2011). 
Es dentro de esta problemática que se plantean algunas de las hipótesis y objetivos que permitirán determinar ciertas tendencias en el uso de los recursos líticos disponibles y utilizados en la microrregión del Valle de Hualfín:

- Hipótesis 1: Para los momentos tardíos se observa una minimización del tiempo invertido en las distintas etapas de producción de determinados instrumentos líticos. Esto habría estado relacionado a la mayor importancia de las prácticas productivas en la subsistencia y a la necesidad de invertir más tiempo en otros mecanismos burocráticos.

- Hipótesis 2: La tecnología lítica no estuvo orientada principalmente a obtener diseños confiables ni multifuncionales, propicios respectivamente en situaciones de estrés temporal, sino en la obtención de diseños utilitarios, destinados a brindar soluciones adecuadas e inmediatas.

En este sentido, los diseños evidenciarían muy baja inversión de tiempo a lo largo de su producción y uso, y presentarían, entre otras características, baja o nula formatización ${ }^{1}$ de sus filos, baja multifuncionalidad, escasas tareas de mantenimiento y reparación y corta vida útil (Escola 2000, 2004).

Por otro lado, debido a esta baja inversión de energía en el proceso de producción lítica, los grupos tardíos habrían priorizado el aprovechamiento de rocas disponibles en las inmediaciones de los sitios. Por ello sería esperable una alta frecuencia de materias primas muy inmediatas e inmediatas (Civalero y Franco 2003; Elías 2012).

Asimismo, la frecuencia de acceso a otros recursos líticos y minerales disponibles en distintos microambientes de la región, habrían disminuido como resultado de una mayor permanencia en un área determinada debido a la principal actividad de estos grupos (agricultura). Los asentamientos de estas sociedades, basados en una economía agrícola, estarían relacionados a un mayor tiempo de permanencia en estos lugares, y consecuentemente, habrían tenido un menor acceso a otros recursos de mayor trayectoria.

Por lo tanto, en este contexto, nos preguntamos cuáles habrían sido las estrategias y decisiones tecnológicas tomadas por las sociedades tardías que habrían estado influenciadas, posteriormente, por la llegada de los incas en el área de estudio mencionada. Es en este sentido que hemos planteado como objetivo de estudio el análisis de la producción de la tecnología lítica de las sociedades que habitaron el Valle de Hualfín, específicamente del sitio Villavil, en momentos tardíos de ocupación, a partir de la información de base regional de recursos líticos disponibles y de los materiales recuperados en estratigrafía.

\section{Microambiente y recursos líticos disponibles}

El valle de Hualfín se ubica en el sector centro-oeste de la provincia de Catamarca. En la región predomina un clima árido o semiárido, donde las aguas superficiales son muy escasas. Los ríos y arroyos son generalmente de poco caudal y de régimen transitorio. En cambio, las aguas subterráneas pueden ser muy abundantes en el subsuelo

1 Retoque intencional sobre una o ambas caras del borde de una pieza lítica (Aschero 1975, 1983). 


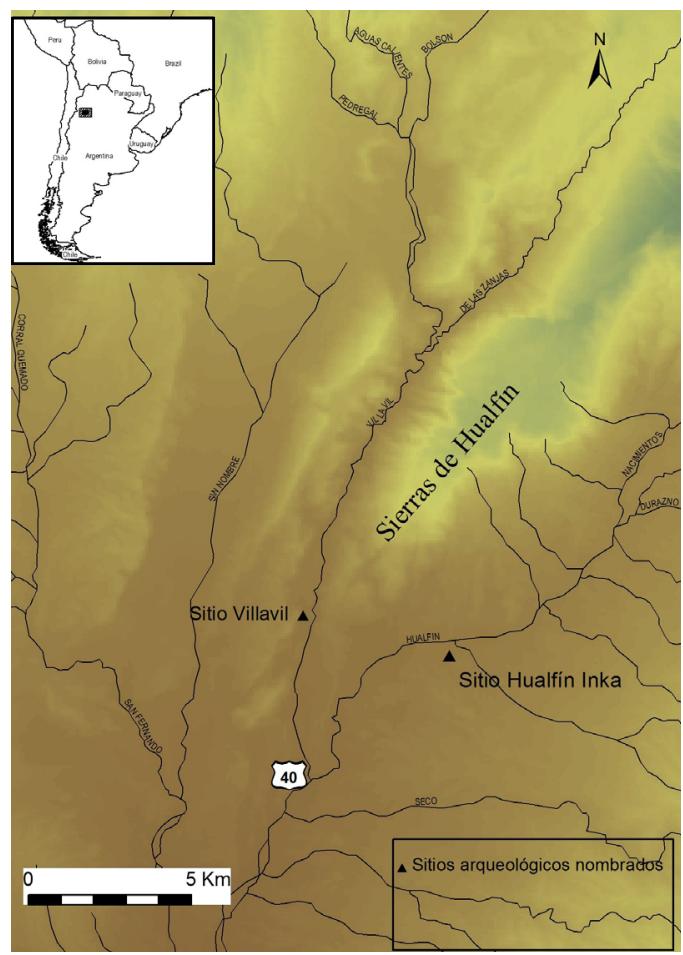

Figura 1: Localización del sitio Villavil, valle de Hualfín, Catamarca.

de los valles y bolsones; los principales acuíferos son los abanicos y llanuras aluviales cuaternarias y aún los sedimentos terciarios (Caminos 1979: 282). En los valles el agente modelador del paisaje es el río, por lo que los depósitos fluviales-aluviales cobran una mayor importancia que los aluviales o coluviales. Tal es el caso del río Hualfín, que presenta en general un curso de poco caudal en los meses de temporada seca (abril-noviembre), aumentando hacia los meses de verano o temporada húmeda (diciembremarzo). Generalmente los depósitos en terrazas son cultivados, capturándose las aguas del río para el riego. También se encuentran aguas termales, relacionadas por lo general con zonas de falla (Caminos 1979: 282). Geológicamente la región se caracteriza por la presencia de las Sierras Pampeanas Noroccidentales con una altura máxima de 3.500 m. El basamento de las mismas está formado por metamorfitas, granitos y roca de mezcla de edad precámbrica a paleozoica inferior. En la faja oriental, que comprende las sierras del valle de Hualfín, este basamento cristalino está compuesto principalmente por rocas metamórficas con un origen basado generalmente en la arena arcillosa: pizarras, filitas y micacitas (Caminos 1979: 282). Por otra parte, en esta faja abundan las rocas de composición granítica y rocas de origen volcánico como el basalto, la dacita y andesitas basálticas, todas ellas originadas de los depósitos del Complejo Volcánico Farallón Negro (Sasso y Clark 1998; «Complejo Volcánico» para González Bonorino 1950; Galli et al. 2012).

\section{Sitio Villavil: características principales}

El sitio Villavil se encuentra ubicado al norte del Valle de Hualfín, entre las coordenadas $66^{\circ} 21^{\prime} 58^{\prime \prime}$ de longitud oeste y $27^{\circ} 13^{\prime} 5^{\prime \prime}$ de latitud sur, sobre una meseta aluvial del río Villa Vil a 1.850 m.s.n.m (Figura 1).

Los trabajos de investigación en el mismo se vienen desarrollando desde el año 2011, habiendo realizado tanto actividades de excavación como de prospección. Asimismo se realizaron trabajos de levantamiento del sitio, ya que se trata de un yacimiento no registrado anteriormente. 
Figura 2: Plano del sitio Villavil confeccionado a partir de estación total (Lynch y Corrado 2014).

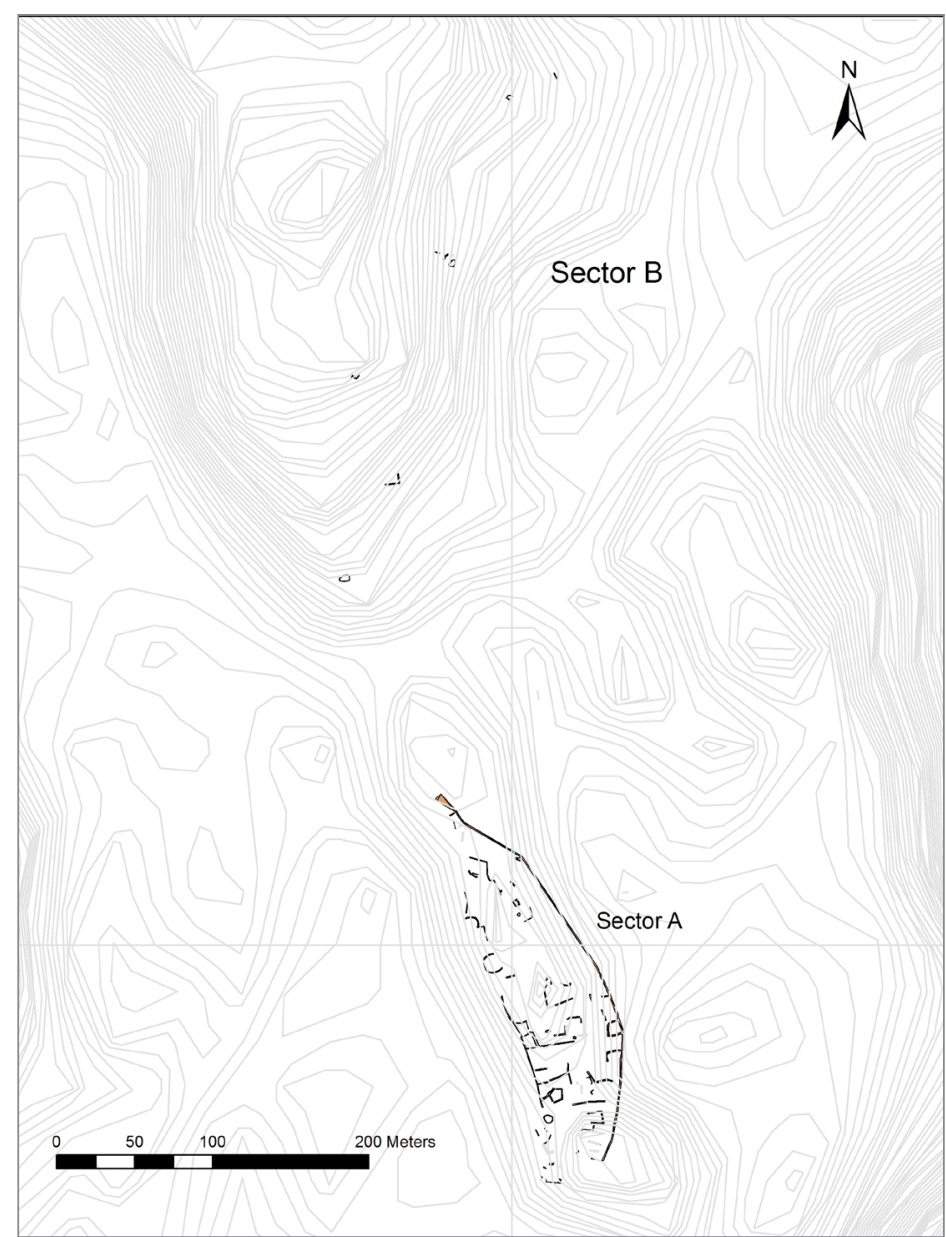

Ha sido dividido metodológicamente en dos sectores, A y B, encontrándose en ambos sectores recintos rectangulares, cuadrangulares y, en menor cantidad, circulares (Figura 2). Los recintos identificados presentan muros de rocas, algunos de una sola hilera, otros dobles sin relleno y dobles con relleno. En superficie se registró una gran cantidad de material cerámico y lítico. Por otra parte, en el sector A se evidenció una mayor cantidad de recintos junto con material superficial, mientras que en el B solo se registraron seis recintos y muy poco material superficial.

La muestra del material lítico analizado proviene de la excavación de uno de los recintos que componen el sitio Villavil: el Recinto Rectangular 1 (Rec. Rec. 1), un recinto rectangular $(4,10 \times 3,80 \mathrm{~m})$ de muros dobles sin relleno, conformado por rocas graníticas y granodioríticas, que resalta del resto de los recintos encontrados en el Sector A por la altura de sus muros, siendo los mismos de $0,80 \mathrm{~m}$ (Lynch 2014: 27). Se excavaron cuatro cuadrículas abarcando la totalidad del recinto, y se plantearon nueve niveles artificiales de excavación de $0,05 \mathrm{~m}$ de espesor, llegando a una 
profundidad de $0,55 \mathrm{~m}$. Dentro de los materiales recuperados se incluyen fragmentos cerámicos atribuibles a los estilos tardíos Santamarianos y de momentos incaicos, así como para el período Temprano o Temprano/Medio como el gris pulido, ante pulido y el gris grabado, algunos pertenecientes al estilo Aguada. Este tipo de registro dentro del conjunto cerámico que incluye estilos tempranos y tardíos se repite en superficie, por lo que podría tratarse de una reocupación del sitio para momentos tardíos (Lynch 2014: 31). Esto último se verificó a través de las dataciones radiocarbónicas obtenidas de material de combustión (fogones) localizado dentro del recinto. Se obtuvieron tres dataciones radiocarbónicas, siendo una de ellas, más antigua, del período Temprano (2190 \pm 80 AP, LP-2979), mientras que las otras dos se ubican en el período Medio/

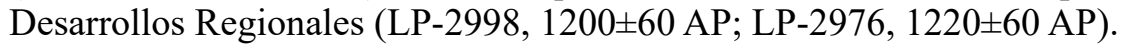

\section{Metodología aplicada}

Como se dijo anteriormente, los materiales analizados fueron obtenidos del Rec. Rec. 1 , siendo el nivel 2 el que presentó mayor cantidad de materiales recuperados, mientras que los niveles 7 y 8 no presentaron evidencia alguna de ocupación.

El análisis en laboratorio abarcó estudios macroscópicos o a simple vista, como también bajo lupa binocular (Nikon SMZ 745, con aumento de 10X a 50X), con el fin de observar las estrías ocasionadas en litos no modificados con posible uso antrópico e identificados como pulidores y/o percutores (Aschero 1975, 1983).

El estudio llevado a cabo sobre los artefactos líticos se realizó a partir de la selección de una serie de variables (tecno-morfológicas) de acuerdo con la propuesta descriptiva de Aschero $(1975,1983)$. Los atributos considerados en el análisis incluyeron:

- Artefactos formatizados: grupo tipológico, materia prima y medidas absolutas.

- Núcleos: materia prima, dimensiones absolutas, porcentaje de corteza y designación morfológica.

- Desechos de talla: estado, materia prima y porcentaje de corteza.

- Artefactos no formatizados: dimensiones absolutas, tipo de lasca, estado, tamaño, módulo de longitud-anchura y anchura-espesor, forma del talón y cantidad de corteza.

- Litos no modificados con evidencia de uso antrópico.

\section{Análisis del material lítico recuperado en el Recinto Rectangular 1}

Entre los materiales recuperados en estratigrafía $(n=129)$, los grupos identificados fueron: artefactos con formatización $(\mathrm{n}=1,1 \%)$, artefactos no formatizados $(\mathrm{n}=62$, $48 \%)$, desechos de talla $(\mathrm{n}=43,33 \%)$, núcleos $(\mathrm{n}=8,6 \%)$ y litos no modificados con posible uso antrópico $(\mathrm{n}=15,12 \%)$. La distribución de las piezas a lo largo de esta estratigrafía constató que el Nivel 2 es el que presenta una mayor concentración de materiales líticos $(n=67,38 \%)$, seguido por los Niveles 5 y $9(n=30,17 \%$ y $n=27$, $16 \%$ respectivamente), mientras que las demás unidades presentan una menor cantidad de ítems (Cuadro 1). 
Cuadro 1: Categorías tipológicas identificadas en los diferentes niveles de excavación del Recinto Rectangular 1 de Villavil

\begin{tabular}{|c|c|c|c|c|c|c|}
\hline Nivel de excavación & 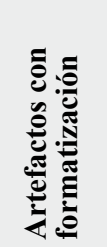 & 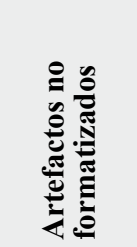 & $\begin{array}{l}\frac{\tilde{a}}{0} \\
\frac{0}{z}\end{array}$ & 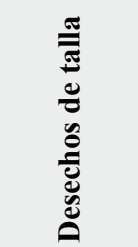 & 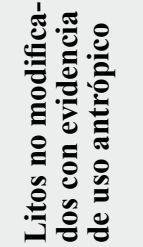 & Total \\
\hline Nivel 1 & - & 10 & 1 & - & 2 & $13(9 \%)$ \\
\hline Nivel 2 & - & 22 & 5 & 18 & 2 & $47(38 \%)$ \\
\hline Nivel 3 & 1 & 6 & 1 & 5 & 2 & $15(12 \%)$ \\
\hline Nivel 4 & - & - & - & 2 & - & $2(2 \%)$ \\
\hline Nivel 5 & - & 16 & 1 & 7 & 5 & $30(17 \%)$ \\
\hline Nivel 6 & - & 3 & - & 4 & - & $7(6 \%)$ \\
\hline Nivel 9 & - & 5 & - & 7 & 4 & $16(16 \%)$ \\
\hline Total & $1(1 \%)$ & $62(48 \%)$ & $8(6 \%)$ & $43(33 \%)$ & $15(12 \%)$ & $129(100 \%)$ \\
\hline
\end{tabular}

Figura 3: Artefacto formatizado (B) recuperado en estratigrafía y material cerámico asociado (A).
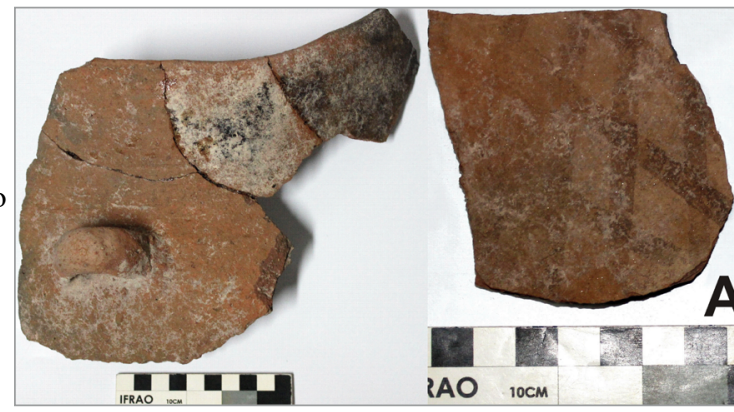

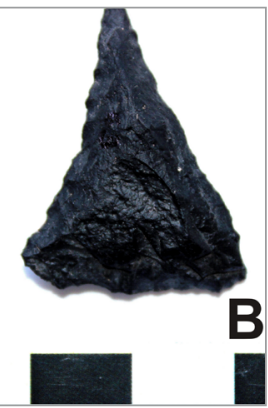

Del análisis del conjunto material se observó que la mayor parte corresponde a elementos cerámicos con un bajo grado de fragmentación, sin embargo la presencia de abundante material lítico ha permitido realizar un análisis más detallado del conjunto recuperado.

En relación con la tecnología lítica, podemos mencionar que el grupo de los artefactos formatizados se encuentra representado por una única pieza que corresponde al grupo tipológico de perforadores (Aschero 1975, 1983), elaborado sobre basalto de buena calidad para la talla, de coloración negra y de $46 \mathrm{~mm}$ de largo, 62,1 $\mathrm{mm}$ de ancho y $10 \mathrm{~mm}$ de espesor (Figura 3B). El resto de la producción lítica recuperada en estratigrafía corresponde a núcleos, desechos de talla y artefactos sin formatización.

\subsection{Núcleos}

Dentro del conjunto analizado se identificaron 8 núcleos que en su mayoría correspondían a diferentes variedades de basalto como materia prima, a excepción de un único elemento que fue reconocido como sílex. El mayor número de núcleos fue recuperado del Nivel 2 de la secuencia $(n=5,62 \%)$, mientras que en los demás niveles se dispusieron en menores cantidades $(n=1,13 \%)$. Varios de estos núcleos se 


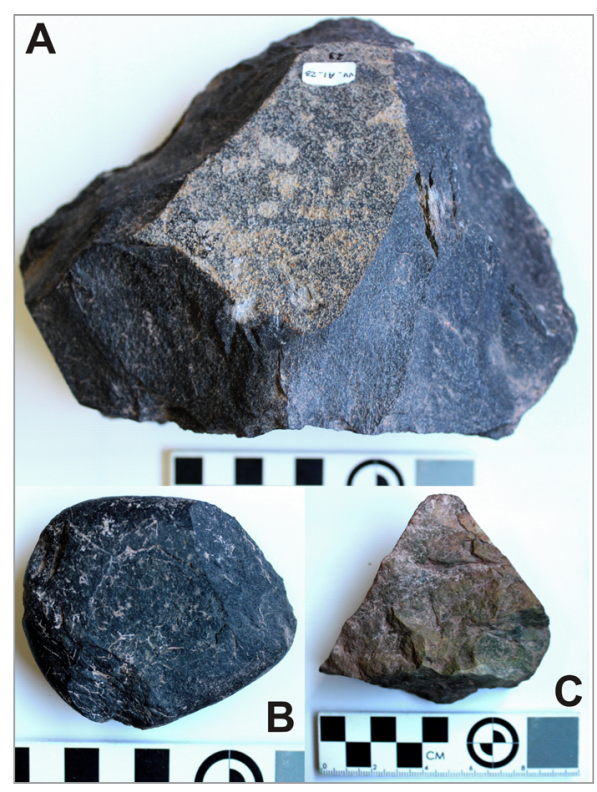

Figura 4: Núcleos identificados en el Recinto Rectangular 1 del sitio Villavil. A) Núcleo de basalto no agotado y con potencialidad de extracción. B) Guijarro con extracciones aisladas. C) Núcleo de sílex marrón-verdoso con lascados aislados.

encontraban fracturados, sin embargo se pudo identificar que los de morfología con lascados aislados fueron los más recurrentes en el conjunto analizado, a excepción de un único caso definido como poliédrico (Aschero 1975,1983 ).

En el Nivel 2, además de presentar el mayor número de estos elementos, se recuperó el núcleo de mayor volumen en toda la secuencia $\left(1.763 .916 \mathrm{~mm}^{3}\right)$. Este correspondía a un basalto pero de mejor calidad que los identificados en este mismo nivel (Figura 4). El núcleo con menor volumen de la secuencia fue identificado en los niveles más profundos $\left(33.045,18 \mathrm{~mm}^{3}\right)$ e igualmente correspondía a un basalto, pero de menor calidad. Este núcleo a su vez presentó el mayor número de extracción en toda la secuencia, identificándose 10 extracciones sobre el mismo. En este sentido, se cree que la masa inicial del mismo habría sido de mayor tamaño que el indicado anteriormente, aun conservando la presencia de corteza y por lo tanto, potencialidad de extracción.

En cuanto al resto de los núcleos recuperados se reconoció un promedio de 6 extracciones por elemento; sin embargo, se observó cierta relación entre la calidad de la materia prima seleccionada y la cantidad de extracciones presentes. Los núcleos de basalto con menos impurezas y, por lo tanto, considerados como una materia prima homogénea y de mejor calidad para la talla, fueron aquellos que presentaron una mayor cantidad de extracciones, sin embargo no se pudo reconocer diferencias en cuanto al largo máximo de los lascados. Los núcleos de basalto de menor calidad presentaron lascados de dimensiones semejantes o en algunos mayores $\left(4.077 \mathrm{~mm}^{2}\right)$ al de aquellos de mejor calidad $\left(1.428 \mathrm{~mm}^{2}\right)$, siendo en este caso consecuencia probable de las diferentes etapas de reducción del núcleo. A diferencia de estos basaltos, en aquellos de calidad regular o intermedia, se cree que podrían haber incluido únicamente las etapas de descortezamiento de estos núcleos, debido a la presencia de impurezas que habrían dificultado la tarea de extracción y, por lo tanto, la obtención de lascas primarias o secundarias exclusivamente. Aun así, los trabajos de remontaje a futuro permitirán dar más sustento a estas propuestas.

\subsection{Desechos de talla}

Los desechos de talla recuperados de la secuencia constituyen un total de 43 elementos. La mayor cantidad de estos elementos fueron recuperados del Nivel 2 ( $\mathrm{n}=18$, 
$42 \%$ ), sin embargo altos porcentajes de estos desechos fueron identificados, igualmente, en los Niveles 3,5 y $9(n=5,11 \% ; n=7,16 \%$ y $n=5,12 \%$ respectivamente).

En cuanto a su estado se reconoció un alto porcentaje de piezas fracturadas $\sin$ talón $(\mathrm{n}=24,56 \%) \mathrm{y}$, en menores proporciones, enteras $(\mathrm{n}=12,28 \%)$, fracturadas con talón $(\mathrm{n}=6,14 \%)$ e indeterminadas $(\mathrm{n}=1,2 \%)$.

En cuanto a la materias primas utilizadas en su producción se reconoció, al igual que entre los artefactos no formatizados, altos porcentajes del uso de basalto $(n=27$, $63 \%$ ), materia prima de origen local y con abundante disponibilidad en lugares cercanos al sitio. En menores proporciones se identificó el uso de sílex $(\mathrm{n}=4,9 \%)$, dacita $(n=3,7 \%)$ y otras rocas que no alcanzan el $5 \%$ de la muestra, como calcedonia, granito y andesita.

Teniendo en cuenta las piezas enteras, el tamaño más frecuente de estos desechos corresponde a piezas pequeñas $(\mathrm{n}=4,34 \%)$ y mediano-pequeñas $(\mathrm{n}=4,33 \%)$, mientras que el módulo de longitud-anchura más recurrente corresponde al mediano normal $(\mathrm{n}=6,50 \%)$.

Por otro lado, respecto a la cantidad de corteza que presentaron estos desechos, se observó un alto porcentaje de piezas sin corteza $(n=22,54 \%)$. En este sentido y debido a la escasa presencia de artefactos formatizados en estratigrafía, se cree que estos bajos porcentajes estarían relacionados con las diferentes etapas de reducción de los núcleos, actividad que habría sido realizada en el interior del sitio.

\subsection{Artefactos no formatizados}

De este grupo fueron identificados un total de 62 elementos, dentro de los cuales el mayor número de piezas fue recuperado en el Nivel $2(n=22,35 \%)$. El total presentó un alto porcentaje de elementos fracturados sin talón $(n=26,42 \%)$, seguido de menores proporciones de piezas enteras $(n=20,32 \%)$ y fracturadas con talón $(n=16,26 \%)$. Asimismo, una baja proporción de estas piezas se vieron afectadas por la presencia de pátinas blancas y depositaciones de concreciones carbonáticas propias del medio en el que fueron depositadas y que, en algunos casos, ha dificultado la identificación y el reconocimiento de ciertos rasgos tecnológicos. Aún así, en aquellas piezas donde estas alteraciones se hicieron presentes, el grado de alteración no fue tan intenso permitiendo identificar los principales rasgos considerados en el análisis, al igual que la determinación de diferentes tipos de artefactos.

Por lo tanto, a partir del análisis realizado se reconoció que las materias primas utilizadas en su producción incluyeron rocas de origen autóctono mayoritariamente ígneas (González Bonorino 1950, 1951; Muruaga 2001:19-20, Da Poian et al. 2014: S22-18).

En cuanto a los tipos de lascas identificas se registró un alto porcentaje de lascas externas [primarias $(n=14,22 \%)$ y secundarias $(n=14,22 \%)$ ], al igual que internas con una proporción mayor de lascas angulares $(n=9,14 \%)$. Sin embargo, también se registraron menores cantidades de lascas de módulo laminar $(\mathrm{n}=6,8 \%)$ y la presencia de guijarros $(7 \%)$, los cuales podrían haber sido utilizados como formas bases en la producción de artefactos formatizados o a partir de sus filos naturales (Cuadro 2). 
Cuadro 2: Tipo de lascas registradas en el conjunto analizado del sitio Villavil, recuperadas del Recinto Rectangular 1

\begin{tabular}{lcc}
\hline \multicolumn{1}{c}{ Tipo de lascas } & Frecuencia & Porcentaje \\
\hline Guijarro de sección elíptica & 2 & 3 \\
Guijarro plano convexo & 1 & 2 \\
Nódulo tabular & 1 & 2 \\
Lasca primaria & 20 & 32 \\
Lasca secundaria & 14 & 22 \\
Lasca con dorso & 3 & 5 \\
Lasca angular & 9 & 14 \\
Lasca de arista & 2 & 3 \\
Lasca tableta de núcleo & 1 & 2 \\
Lasca indiferenciada & 4 & 6 \\
Lasca de módulo laminar & 5 & 8 \\
Total & 62 & 100 \\
\hline
\end{tabular}

En relación con la presencia de talones en su mayoría corresponden a diedros $(\mathrm{n}=13,41 \%)$, seguido de lisos naturales $(\mathrm{n}=11,34 \%)$ y en menores proporciones liso $(\mathrm{n}=7,22 \%)$ y poliedros $(\mathrm{n}=1,3 \%)$. Asimismo, la mayoría de la piezas no presentaron bulbos bien definidos ( $\mathrm{n}=37,62 \%)$, lo que podría indicar que los percutores utilizados durante el proceso de talla hayan sido elaborados de material con dureza intermedia (hueso o madera). Sin embargo, no se descarta el uso de percutores duros, dado que han sido igualmente recuperados en estratigrafía y con marcas de impacto (ver Figura 7 A).

Por otro lado, respecto a las dimensiones de las piezas enteras analizadas, la mayor cantidad de ANF presentaron tamaños mediano-grandes $[(\mathrm{n}=9,45 \%)$, con valores cercanos a $38 \mathrm{~mm}$ de largo, $29 \mathrm{~mm}$ de ancho y $11 \mathrm{~mm}$ de espesor]. La diferencia con respecto a las proporciones del tamaño grande no fue significativa $[(\mathrm{n}=8,40 \%)$, con valores cercanos a $51 \mathrm{~mm}$ de largo, $48 \mathrm{~mm}$ de ancho, $17,5 \mathrm{~mm}$ de espesor] (Figura $5 \mathrm{~A})$; mientras que una menor cantidad de piezas se incluyeron en la categoría muygrande $[(\mathrm{n}=3,10 \%), 80 \mathrm{~mm}$ de largo, $72 \mathrm{~mm}$ de ancho y $35 \mathrm{~mm}$ de espesor] (Figura $5 \mathrm{~B})$.

En cuanto al módulo de longitud-anchura la mayoría de las piezas presentaron módulo corto ancho $(\mathrm{n}=7,35 \%)$, mientras que en menor medida mediano normal

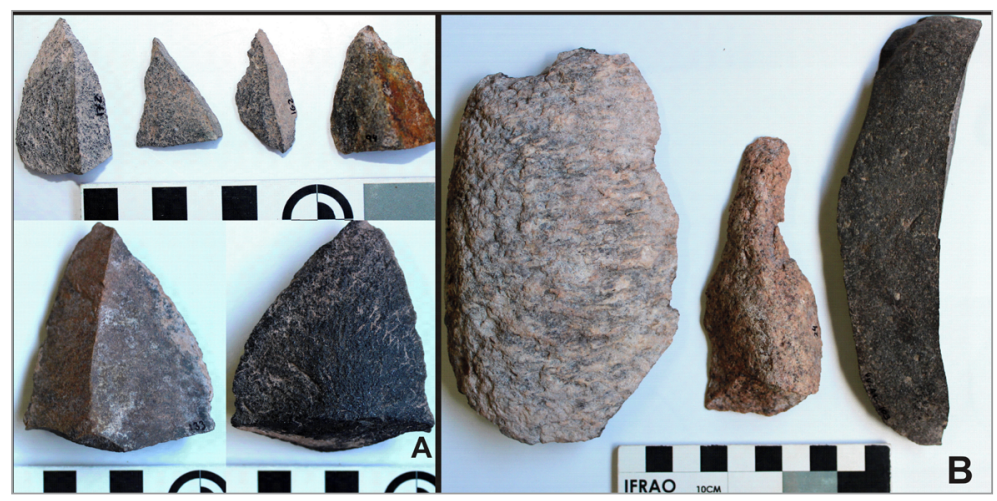

Figura 5: Artefactos sin formatización. A) Lascas internas de diferentes morfologías.

B) Lascas primarias de tamaño muy grande, elaboradas sobre materia prima local (granito y basalto de buena calidad). 
$(\mathrm{n}=5,25 \%)$ y mediano alargado $(\mathrm{n}=4,20 \%)$, y en menores e iguales proporciones se localizan los módulos laminares angosto, laminar normal, corto muy ancho y corto anchísimo con un único elemento en cada caso (5\%).

En relación con el espesor, la mayoría de las piezas analizadas presentaron módulos muy espesos $(\mathrm{n}=31,51 \%$, con valores cercanos a $18 \mathrm{~mm})$, esto probablemente sea consecuencia del alto porcentaje de lascas primarias, secundarias y angulares que constituyeron los mayores porcentajes dentro de la muestra analizada $(32 \%, 22 \%$, $14 \%$ respectivamente).

\subsection{Litos no modificados con evidencia de uso antrópico}

Dentro de esta categoría se han incluido aquellas piezas que presentan modificaciones sobre su superficie a partir de la presencia de rastros (estrías registradas a simple vista o bajo una lupa binocular) que son interpretadas como consecuencia de su posible uso antrópico. Aún así, futuros análisis permitirán profundizar los conocimientos adquiridos respecto a esta categoría, por lo que en este trabajo sólo se menciona su presencia $\mathrm{y}$, a grandes rasgos, se describen las características principales de los mismos.

El total de litos no modificados con posible evidencia de uso incluye 15 elementos, de los cuales la mayor cantidad ha sido recuperado del Nivel 5 de la secuencia estratigráfica $(n=5,34 \%)$, seguido por el Nivel 9 del cual fueron recuperados 4 elementos $(27 \%)$.

En cuanto a las materias primas identificadas se pudo reconocer, al igual que en el restos de los materiales, un mayor uso de basalto $(n=11,73 \%)$ seguido de menores proporciones de otras variedades de materias primas como riolita (13\%), pizarra y granito ( $7 \%$ respectivamente).

Varios de estos elementos presentaron estrías paralelas entre sí, lo que podría ser consecuencia del contacto de estas piezas con material duro de manera transversal (Figuras 6 y 7). Sin embargo, hasta el momento la funcionalidad para la cual habrían sido destinados estos elementos son inciertas, aunque la hipótesis más sostenida a partir de la observación de otras colecciones recuperadas en sectores cercanos al sitio estudiado, permitiría proponer su intervención en la manufactura cerámica como pulidores, para el acabado y decorado de las piezas.

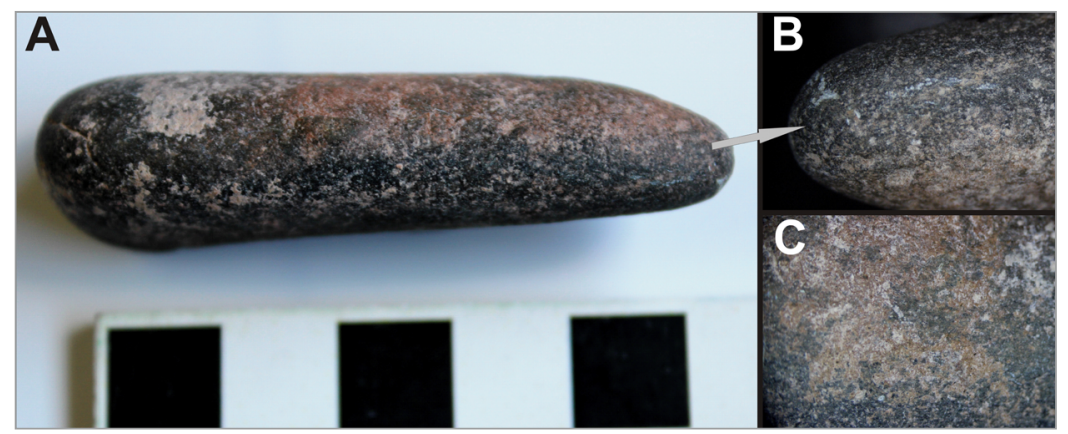

Figura 6: A) Lito no modificado con evidencia de uso antrópico. B) Fotomicrografía de estrías identificadas sobre la superficie de estos elementos (aumento 10X). C) Restos de residuo identificado sobre su superficie, posible pigmento de coloración rojo (aumento 10X). 


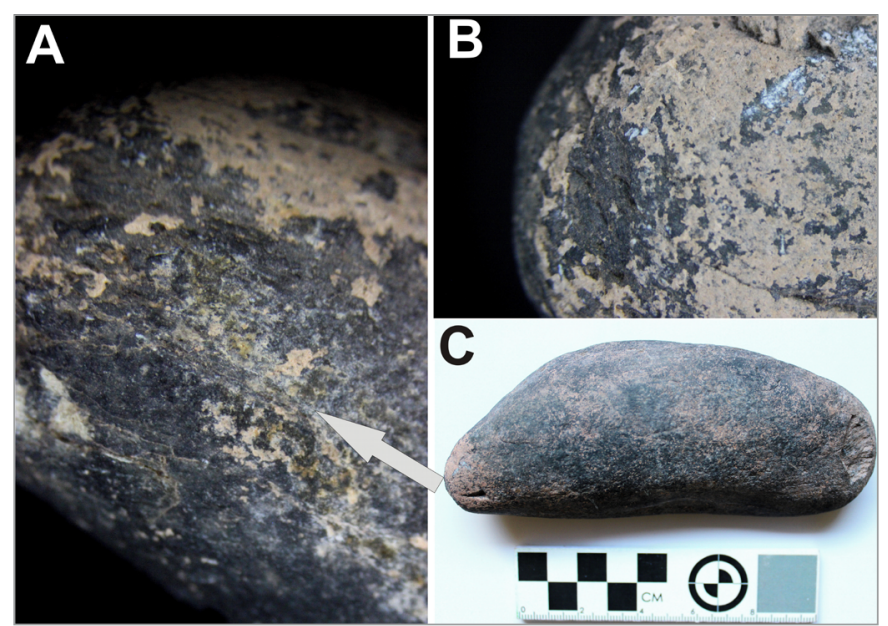

Figura 7: A) Estrías identificadas por potencial uso. B) Microlascados y puntos de impacto ocasionados por uso. C) Percutor identificado en la secuencia estratigráfica de Villavil.

\section{Discusión y conclusiones}

Los resultados presentados en este trabajo han permitido incrementar la información que se tiene de las poblaciones locales que habitaron el sitio Villavil; específicamente respecto a la relación que mantuvieron con su entorno y a las estrategias aplicadas por parte de estos grupos sedentarios, en la obtención y utilización de recursos líticos disponibles para la producción de diferentes tecnologías.

En cuanto al aprovisionamiento de materias primas se evidencian dos tendencias en el área de estudio y sectores cercanos. Por un lado, se nota un predominio de rocas locales donde se manifiesta una baja inversión de tiempo y cuidado en la elaboración artefactual (Lynch 2010; Lynch y Lynch 2011; Flores 2012), mientras que en las foráneas, principalmente obsidianas, existe una mayor inversión en el tiempo de manufactura para determinados artefactos, como las puntas de proyectil (Flores y Wynveldt 2009)

En el caso del sitio Villavil las materias primas identificadas a partir de los materiales analizados, corresponden en su mayoría a rocas de procedencia local (a una distancia menor de $40 \mathrm{~km}$ ), con altos porcentajes de basaltos de buena calidad para la talla. Estos habrían sido obtenidos de fuentes secundarias en forma de cantos rodados que se encuentran en el margen del río que atraviesa el sitio. Por otro lado, la presencia de guijarros, como potenciales formas bases y de buena calidad para la talla, permitiría plantear que los nódulos habrían sido cuidadosamente seleccionados atendiendo a sus atributos para la obtención de productos capaces de cumplir diferentes actividades a partir de sus filos naturales. Estas observaciones estarían corroborando la hipótesis 1 presentada al inicio de este trabajo.

Por otra parte, la presencia de núcleos hacia el interior del recinto, en su mayoría de lascados aislados, estaría indicando una morfología no estandarizada y por lo tanto un carácter irregular de las extracciones.

En cuanto a las etapas de reducción, principalmente respecto al uso del basalto, varias se verían reflejadas en el registro. Pudieron ser recuperados núcleos (en su 
mayoría de lascados aislados), percutores con puntos de impacto y altos porcentajes de lascas primarias y secundarias, al igual que desechos de talla sobre este tipo de materia prima.

En cuanto a los productos de talla recuperados se identificaron altos porcentajes de lascas como formas bases (principalmente primarias, secundarias y angulares). Sin embargo, también se habrían producido artefactos sin formatización de módulo laminar, generando filos largos y rectos capaces de cumplir de manera efectiva y sin modificación previa, una amplia diversidad de funciones. En cuanto a las materias primas identificadas en su producción, al igual que en el resto de los materiales, se observaron altos porcentajes de uso de basalto. Pero se registraron proporciones menores de otras materias primas como granito, dacita y sílice (6\% y 5\% respectivamente). Su procedencia, al igual que el basalto, sería local y, probablemente, en forma de cantos rodados que habrían sido traídos al sitio debido a sus características propicias para la talla.

A su vez se registró una baja producción de artefactos formatizados, representados por una única pieza (del grupo de perforadores), indicando, una baja inversión de tiempo y energía de trabajo en la producción tecnológica. Otros autores ya habrían señalado esto para el sector de Antofagasta de la Sierra en momentos Tardío e Inka (Elías 2012). Las puntas de proyectil y los perforadores, artefactos elaborados para tareas específicas, habrían presentado una mayor inversión de esfuerzo en su manufactura respondiendo a estrategias de tipo conservativas (Elías 2007). Por otro lado, los trabajos publicados de Parry y Kelly (1987), afirmaron que los grupos sedentarios pueden apilar materias primas en sus bases residenciales, reducir su producción de artefactos formales y utilizar artefactos expeditivos. En este sentido, gran parte del conjunto lítico analizado habría sido resultado de decisiones y estrategias expeditivas, conformando diseños utilitarios convalidando la hipótesis 2 (Escola 2000).

A su vez, la identificación de otros elementos que formaron parte de esta tecnología lítica, como el caso de litos no modificados con posible evidencia de uso antrópico, constituyen parte de la cultura material recuperada del registro bajo estudio, y se cree que los mismos han sido de fundamental importancia como parte utilitaria en la manufactura de la cerámica, que habría sido desarrollada en el sitio. La producción cerámica de características de uso doméstico permitiría, sumado al análisis de la tecnología lítica, formular algunas interpretaciones acerca de la funcionalidad que habría tenido este recinto en momentos tardíos.

Por lo tanto, de acuerdo con los resultados obtenidos del análisis de estos materiales, se podría plantear que, si bien el recinto evidencia una ocupación temprana, podría tratarse de una estructura habitacional o haber funcionado como un lugar de almacenamiento, debido a la gran cantidad de fragmentos cerámicos que podrían haber sido utilizados como contenedores. Por otra parte, tanto en superficie como en estratigrafía, se han registrado fragmentos de cerámica incaica, lo que estaría indicando una ocupación prolongada hasta la llegada del Imperio en el valle (Lynch 2014: 494). A partir de las investigaciones realizadas con anterioridad en la zona de estudio, como los sitios Hualfín Inka (Lynch 2010, Lynch y Lynch 2011, Lynch et al. 2013), El Shincal y Los Colorados (Giovannetti 2009), sumado a los últimos trabajos realizados en Villavil (Lynch 2014), se podría plantear que, para el momento de 
la llegada incaica a la región, se habría instaurado un tipo de relación indirecta con las poblaciones locales, en donde las relaciones de alianza y de reciprocidad, dentro de la gran variabilidad de las estrategias políticas estatales, habrían sido sumamente importantes.

Si bien hasta el momento se sigue trabajando en el sitio Villavil, y se debe profundizar aún más en análisis específicos y nuevas excavaciones que permitan sostener los postulados acerca de su posible funcionalidad, se podría plantear que el sitio habría sido ocupado por poblaciones agropastoriles desde tiempos tempranos, con una reocupación en momentos más tardíos. El mismo habría funcionado como un asentamiento en donde sus ocupantes habrían desarrollado actividades cotidianas y que, al momento de la llegada del inca, tuvieron que modificar su organización social, política y económica a partir del nuevo régimen instaurado.

Si bien este trabajo constituye una primera aproximación a la tecnología lítica del sitio Villavil, la interpretación que se ha hecho permite explorar nuevos espacios y esbozar nuevas hipótesis interpretativas en relación con las dinámicas pasadas de las comunidades prehispánicas, tanto para momentos previos como posteriores a la llegada del inca y su nuevo orden social.

Agradecimientos: A CONICET y a la Agencia Nacional de Promoción Científica y Tecnológica por el financiamiento de los trabajos realizados, a Emiliano Bentivenga y Milagros Ríos Malán por los trabajos realizados tanto en el campo como en el laboratorio. Todo lo escrito aquí es exclusiva responsabilidad de los autores.

\section{Referencias Bibliográficas}

Aschero, Carlos

1975 Ensayo para una clasificación morfológica de artefactos líticos aplicada a estudios tipológicos comparativos. Manuscrito. Buenos Aires: Informe al CONICET.

1983 Ensayo para una clasificación morfológica de artefactos líticos. Apéndices A-C. Manuscrito. Buenos Aires: Cátedra de Ergología y Tecnología. Facultad de Filosofía y Letras, Universidad de Buenos Aires.

Ávalos, Julio César

1998 «Modos de uso de implementos agrícolas de la Quebrada de Humahuaca y Puna a través del análisis de huellas de desgaste», en Los desarrollos locales y sus territorios. Arqueología del NOA y sur de Bolivia, $\mathrm{M}^{\mathrm{a}}$ Beatriz Cremonte, comp., pp. 285-303. San Salvador de Jujuy: Universidad Nacional de Jujuy.

2002 Sistema de producción lítica de una comunidad tardía de la quebrada de Humahuaca. Tesis Licenciatura. Facultad de Humanidades y Ciencias Sociales, Universidad Nacional de Jujuy.

Ávalos, Julio César y María Gabriela Chaparro

2008 «Los artefactos líticos durante la ocupación inka en la quebrada de Humahuaca: producción y circulación», en Problemáticas de la Arqueología Contemporánea, Actas del XV Congreso Nacional de Arqueología Argentina, Tomo II, A. Austral y M. Tamagnini, comps., pp. 473-474. Córdoba: Publicaciones de la Universidad Nacional de Río Cuarto. 
Caminos, Roberto

1979 «Sierras Pampeanas Noroccidentales, Salta, Tucumán, Catamarca, La Rioja y San Juan », en Segundo Simposio de Geología Regional Argentina 1, A.F. Leanza, ed., pp. 225-282. Córdoba: Academia Nacional de Ciencias.

Chaparro, María Gabriela

2002 «Informe de Análisis Lítico del Sitio Tolombón, Provincia de Salta. Trabajo de campo año 2001». Intersecciones en Antropología 3: 119-123.

2009 «El manejo de los recursos líticos en el pasado. Sociedades pre-estatales y estatales en el Área Valliserrana del Noroeste argentino (1000-1536 DC)». Tesis de Doctorado. Buenos Aires: Facultad de Filosofía y Letras, Universidad de Buenos Aires.

2013 «Preferencias en el manejo cotidiano de rocas. Los artefactos líticos de los asentamientos estatales del sur de la Quebrada de Humahuaca y el valle Calchaquí medio (Argentina)», en Al Borde del Imperio. Paisajes sociales en áreas periféricas del Qollasuyu, V. Williams y M. B. Cremonte, eds., pp. 273-309. Buenos Aires: Universidad de Buenos Aires.

Chaparro, María Gabriela y Julio César Ávalos

2014 «La tecnología lítica durante la ocupación Inka en la Quebrada de Humahuaca (Provincia de Jujuy, Argentina)», en Artefactos líticos, movilidad y funcionalidad de sitios: problemas y perspectivas, Patricia S. Escola y Salomón Hocsman, comp., pp. 59-68. Oxford: BAR, Archaeopress.

Civalero, María Teresa y Nora Franco

2003 «Early human occupations in Western Santa Cruz Province, Southernmost South America». Quaternary International 109-110: 77-86.

Da Poian, Gabriela, Raúl Seggiaro, Florencia Bechis y Víctor Hugo García

2014 «Cinemática y edad de la estructuración compresiva neógena de la Sierra de Hualfín, Provincia de Catamarca». Ponencia presentada en XIX Congreso Geológico Argentino, Córdoba. Asociación Geológica Argentina.

ELÍAs, Alejandra

2005 «Informalidad: un acercamiento inicial a la tecnología lítica de momentos tardíos a partir de las características de diseño de los instrumentos relevados en dos sitios de Antofagasta de la Sierra (Prov. de Catamarca, Argentina): La Alumbrera y Campo Cortaderas». Hombre y Desierto 12: 47-71.

2007 «Tecnología lítica en las sociedades tardías de Antofagasta de la Sierra (Puna Meridional Argentina)». Estudios Atacameños 33: 59-85.

2008 «Estrategias tecnológicas y variabilidad de los conjuntos líticos de las sociedades de los Períodos Tardío y Tardío-Inka en Antofagasta de la Sierra (Prov. de Catamarca, Puna Meridional argentina) y Doncellas (Prov. de Jujuy, Puna Septentrional argentina)». Comechingonia virtual 1: 43-72.

2012 «Tecnología lítica en el Período Tardío ( $c a$. 1100-550 años AP) de Antofagasta de la Sierra (Provincia de Catamarca, Puna Meridional Argentina)». Relaciones de la Sociedad Argentina de Antropología XXXVII (1): 19-41.

ELÍAs, Alejandra y Patricia Escola

2010 «Viejos y nuevos horizontes: obsidianas entre las sociedades agropastoriles del periodo Tardío en Antofagasta de la Sierra (provincia de Catamarca, puna meridional argentina)». Revista Española de Antropología Americana 40 (2): 9-29. 
Escola, Patricia

2000 Tecnología lítica y sociedades agro-pastoriles tempranas. Tesis Doctoral. Facultad de Filosofía y Letras, Universidad de Buenos Aires.

2004 «Variabilidad en la explotación y distribución de obsidianas en la Puna meridional argentina». Estudios Atacameños 28: 9-24.

FLORES, Marina

2012 «El cuarzo como materia prima en el valle de Hualfín (Provincia de Catamarca, Argentina) durante el período de Desarrollos Regionales/Inka». Comechingonia 16: 297-305.

FLORES, Marina y Federico WYNVELDT

2009 «Análisis tecno-tipológico de los artefactos líticos de la Loma de los Antiguos de Azampay (Departamento de Belén, Catamarca)». Intersecciones en Antropología 10: 221-235.

Galli, Claudia Inés, Pablo Caffe, Marcelo Arnosio, Raúl Seggiaro, y Raúl Becchio

2012 «Análisis paleoambiental y procedencia de los depósitos cenozoicos en el extremo suroeste de la Sierra de Aconquija, Provincia de Catamarca». Revista de la Asociación Geológica Argentina 69 (4): 596-610.

GiovannetTi, Marco Antonio

2009 Articulación entre el sistema agrícola, redes de irrigación y áreas de molienda como medida del grado de ocupación Inka en El Shincal y Los Colorados (prov. de Catamarca). Tesis doctoral. Facultad de Ciencias Naturales y Museo. Universidad Nacional de La Plata.

GonzÁlez Bonorino, Félix

1950 «Algunos problemas geológicos de las Sierras Pampeanas». Revista de la Asociación Geológica Argentina 5 (3): 81-110.

1951 «Una nueva formación Precámbrica en el noroeste Argentino». Comunicaciones Cientificas del Museo de La Plata 5: 4-6.

LEDESMA, Rossana

2003 «Diseño de puntas de proyectil. Una vía de análisis alternativo para el estudio de identidad en la Quebrada del Toro, provincia de Salta, Argentina». Cuadernos de la UNJU 20: 241-269.

LYNCH, Julieta

2010 La construcción del paisaje y la organización del espacio en el sector Norte del Valle de Hualfin, Provincia de Catamarca. Tesis doctoral. Facultad de Ciencias Naturales y Museo de la Universidad Nacional de La Plata.

2014 «Sitio Villavil: una aproximación a la dinámica local-estatal al norte del valle de Hualfín, Catamarca». Revista Intersecciones en Antropología 15 (2): 491-496.

LYNCH, Julieta y Virginia LYNCH

2011 «Hualfín Inka: tecnología lítica de un centro administrativo (Catamarca, Argentina)». Arqueología Iberoamericana 12: 15-27.

Lynch, Julieta, Marco Antonio Giovannetti y María Cecilia PÁEZ

2013 «Ushnus of the Inca provincial region: An analysis of two ceremonial platforms from Inca sites in Catamarca (Argentina)». Journal of Anthropological Archaeology 32 (1): 97-108 
LYNCH, Julieta y Gustavo CORRADO

2014 «Arqueología virtual aplicada al sitio Villavil, Catamarca, Argentina». Virtual Archaeology Review 5 (11): 86-92.

Muruaga, Claudia

2001 «Petrografía y procedencia de areniscas terciarias en la Subcuenca de Hualfín, provincia de Catamarca, noroeste de Argentina». AAS Revista. Asociación Argentina de Sedimentología 8 (2): 15-35.

Nelson, Margaret C.

1991 «The Study of Technological Organization». Archaeological Method and Theory 3: 57-100.

1996 «Technological Strategies Responsive to Subsistence Stress», en Evolving Complexity and Environmental Risk in the Prehistory of Southwest, Joseph Tainter y Bonnie Tainter, eds., pp. 107-143. Santa Fe: Addison-Wesley.

Olivera, Daniel y Mercedes Podestá

1993 «Los recursos del arte: arte rupestre y sistemas de asentamiento-subsistencia formativos en la Puna meridional argentina». Arqueología. Revista de la Sección de Prehistoria 3: 93-141.

Olivera, Daniel y Silvina VigLiani

2000-02 «Proceso cultural, uso del espacio y producción agrícola en la Puna Meridional Argentina». Cuadernos del Instituto Nacional de Antropología y Pensamiento Latinoamericano 19: 459-481.

PARRY, William y Robert KELLY

1987 «Expedient Core Technology and Sedentism», en The Organization of Core Technology. Jay K. Johnson y Carol A. Morrow, eds., pp. 285-304. Boulder: Westview Press.

RATTO, Norma

2003 Estrategias de caza y propiedades del registro arqueológico en la Puna de Chaschuil (Departamento Tinogasta, Catamarca, Argentina). Tesis doctoral. Facultad de Filosofía y Letras, Universidad de Buenos Aires.

RatTo, Norma y Verónica Williams

1995 «Materias primas líticas y procesos de producción en el sitio Potrero-Chaquiago (Catamarca): las apariencias engañan». Relaciones de la Sociedad Argentina de Antropología XX: 141-162.

Sasso, A. M. y A. H. Clark

1998 «The Farallón Negro Group, northwest Argentina: magmatic, hydrothermal and tectonic evolution and implications for $\mathrm{Cu}-\mathrm{Au}$ metallogeny in the Andean backarc». Society of Economic Geologists Newsletter 34: 8-18

SPROVIERI, Marina

2005 Manejo de recursos y producción lítica en sociedades tardias del valle Calchaquí (Salta). Tesis Licenciatura. Facultad de Filosofía y Letras, Universidad de Buenos Aires.

2006 «Circulación de obsidianas en el valle Calchaquí, Salta: análisis por activación neutrónica para los Períodos de Desarrollos Regionales e Inca». Ponencia presentada en VII Jornadas de Jóvenes Investigadores en Ciencias Antropológicas, Instituto Nacional de Antropología y Pensamiento Latinoamericano. Buenos Aires. 
SPROVIERI, Marina y Lidia BALDINI

2007 «Aproximación a la producción lítica en sociedades tardías. El caso de Molinos 1, Valle Calchaquí Central (Salta)». Intersecciones en Antropología 8: 135-147.

TORRENCE, Robin

1989 «Retooling: toward a behavioral theory of stone tools», en Time, Energy, and Stone Tools, Robin Torrence, ed., pp. 57-66. Cambridge: Cambridge University Press.

YACOBACCIO, Hugo

1994 «Biomasa animal y consumo en el Pleistoceno-Holoceno Surandino». Arqueología. Revista de la Sección de Prehistoria 4: 43-71.

Yacobaccio, Hugo, Patricia Escola, Fernando Pereyra, Marisa Lazzari y Michael GLASCOCK

2004 «Quest for ancient routes: obsidian sourcing research in Northwestern Argentina». Journal of Archaeological Science 31 (2): 193-204. 